\title{
Bronchial Washing to Diagnose Smear-Negative Pulmonary Tuberculosis
}

\author{
Jin Young Lee \\ Department of Internal Medicine, College of Medicine, Kosin University, Busan, Republic of Korea \\ Email: rejim@hanmail.net
}

How to cite this paper: Lee, J.Y. (2019) Bronchial Washing to Diagnose SmearNegative Pulmonary Tuberculosis. Journal of Tuberculosis Research, 7, 143-147. https://doi.org/10.4236/jtr.2019.73014

Received: August 22, 2019

Accepted: September 8, 2019

Published: September 11, 2019

Copyright $\odot 2019$ by author(s) and Scientific Research Publishing Inc. This work is licensed under the Creative Commons Attribution International License (CC BY 4.0).

http://creativecommons.org/licenses/by/4.0/

\begin{abstract}
Introduction: Bronchoscopy with bronchial washing is useful for the diagnosis of pulmonary tuberculosis (TB) when sputum smears are negative. However, its indication has not been standardized. Therefore, we conducted a retrospective study to assess the diagnostic value of bronchial washing in patients suspected with pulmonary TB. Methodology: A retrospective analysis was performed on patients diagnosed with pulmonary TB in Kosin university gospel hospital, a tertiary hospital with 969 beds in South Korea, from March 2017 to December 2018. We obtained three serial sputum samples for acid-fast bacilli (AFB) smear and culture, and all patients underwent bronchoscopy with bronchial washing for AFB smear and culture. Results: Fifty-six patients were enrolled in the study. Smear-negative pulmonary TB (SNPT) was diagnosed in $42 / 56(75 \%)$ patients. Among the patients with smear-positive sputum, 14/14 (100\%) showed culture-positive sputum, while smear- and culture-positive bronchial washing were seen in 7/14 (50\%) and $12 / 14(85.7 \%)$ patients, respectively. Among the patients with SNPT, 17/42 (40.47\%) had culture-negative sputum and were diagnosed using the bronchoscopic washing. Conclusions: For patients with smear-negative sputum, it is necessary to perform bronchoscopic washing to increase the diagnostic rate of pulmonary TB. To control the transmission of pulmonary $\mathrm{TB}$, it is necessary to use a fast and accurate examination method.
\end{abstract}

\section{Keywords}

Bronchial Washing, Pulmonary Tuberculosis, Sputum, Smear, Negative

\section{Introduction}

Pulmonary tuberculosis (TB) is a major public health challenge worldwide, with an estimate of 10.4 million new cases and 1.8 million TB-related deaths in 2015 
[1]. The incidence of TB in South Korea, which has an intermediate TB burden, was 77 per 100,000 people in 2016, which is lower than the global average but is the highest among the members of the Organization for Economic Cooperation and Development [2]. Although various diagnostic tests for pulmonary TB have been developed, its definitive diagnosis relies on culturing Mycobacterium tuberculosis from respiratory secretions. The international guidelines recommend obtaining at least two sputum samples from patients suspected with TB to increase the sensitivity for M. tuberculosis [3]. The World Health Organization defines smear-negative pulmonary TB (SNPT) as a case with at least two sputum samples negative for acid fast bacilli (AFB) and either a positive culture for $M$. tuberculosis or the presence of radiographic abnormalities consistent with $\mathrm{TB}$ [4]. Microbiological tests on flexible bronchoscopy are useful to diagnose SNPT, but their indication has not been standardized. The sensitivity in patients clinically suspected with TB varies from $6.5 \%$ to $77 \%$ [5]. Further evaluations of the bronchoscopic findings are needed to generate evidence-based recommendations for the use of bronchoscopy in patients clinically suspected with SNPT. Therefore, the aim of this study was to assess the diagnostic value of bronchial washing in the patients suspected with pulmonary $\mathrm{TB}$ who are unable to expectorate or have smear-negative sputum.

\section{Methodology}

\subsection{Study Population}

This was a retrospective review of the medical records of patients diagnosed with pulmonary TB in Kosin university gospel hospital, a tertiary hospital with 969 beds in South Korea, from March 2017 to December 2018. We included patients with microbiologically confirmed M. tuberculosis during the study period. We obtained three serial sputum samples for the AFB smear and culture, and all patients underwent bronchoscopy with bronchial washing for the AFB smear and culture. Routine bronchoscopic examinations were performed for patients in this study. Patients who were receiving anti-TB treatment before the bronchoscopy were excluded from the study. This study was approved by the Institutional Review Board of Kosin Medical College.

\subsection{Bronchoscopy Procedures}

Bronchoscopy with bronchial washing was performed using the standard technique [6] [7]. Patients were sedated with intravenous midazolam. Under conscious sedation, a bronchoscope (Olympus, Tokyo, Japan) was inserted using the transnasal or transoral approach, and $5-10 \mathrm{~mL}$ of lidocaine, as needed, was sprayed intratracheally. Subsequently, bronchial washing was performed with 10 $\mathrm{mL}$ of warm sterile saline. This procedure was repeated once in the same segment. The retrieved samples were labeled as the first and second samples. Subsequently, they were tested for AFB on smear and culture. In cases of multiple pulmonary infiltrates detected on chest radiography, bronchial washing was 
performed in the segment exhibiting the most severe abnormalities.

\subsection{Microbiological Examination}

Aliquots of specimens were decontaminated with 2\% N-acetyl-L-cysteine-sodium hydroxide. Subsequently, the specimens were stained with auramine-rhodamine fluorescent and Ziehl-Neelsen stains and examined under a microscope. The worked specimens were plated on both solid (Shinyang, Seoul, Korea) and liquid culture media (MGIT 960 system, Becton Dickinson, Sparks, MD, USA). And they were incubated at $36^{\circ} \mathrm{C}$ for six weeks.

\subsection{Analysis}

Comparison between patients with smear-negative sputum and patients with smear-positive sputum was done with the results of sputum culture, AFB smear and culture results of bronchial washing. Among patients with smear-negative sputum for pulmonary tuberculosis, comparison between patients with culture-negative sputum and patients with culture-positive sputum was done with the results of bronchial washing AFB smear and culture.

Comparisons between groups were made using the Fisher's exact test for categorical variables and the Student's $t$-test for continuous variables. $P$-values $<$ 0.05 were considered statistically significant. All statistical analyses were performed using the Statistical Package for the Social Sciences software version 25.0 (IBM Corp., Armonk, NY, USA).

\section{Results}

Fifty-six patients were enrolled in this study. The median age was 61.5 years. The proportions of men and women were $62.5 \%$ and $37.5 \%$ respectively. No patient had a history of TB. SNPT was diagnosed in $42 / 56$ patients (75\%). Pulmonary TB was diagnosed based on AFB smear-positive sputum in 14/56 (25\%) patients. The mean age of the patients with smear-positive sputum and smear-negative sputum was $65.86 \pm 16.874$ years and $60.05 \pm 18.256$ years, respectively, with no significant difference $(P=0.3)$. The proportion of men was higher than that of women among patients with smear-positive sputum, with no significant difference. All patients with smear-positive sputum (14/14, 100\%) showed culture-positive sputum, while 25/42 (59.5\%) patients with smear-negative sputum showed culture-positive sputum, with a significant difference $(P=0.003)$. Bronchial washing tested positive in $7 / 14(50 \%)$ patients with smear-positive sputum and in $5 / 42(11.9 \%)$ patients with smear-negative sputum, which was significantly different $(P=0.006)$. The proportion of culture-positive bronchial washing was $85.7 \%$ in both groups, with no significant difference $(P=0.65)$ (Table 1). Among the patients with SNPT, $17 / 42$ (40.47\%) had culture-negative sputum and were diagnosed using bronchoscopic washing. Among the patients with SNPT, only $2 / 17$ (11.8\%) showed smear-positive bronchial washing, and 15/17 (88.2\%) showed smear-negative bronchial washing with the result of culture-positive bronchial washing (Table 2). 
Table 1. Comparison between patients with smear-negative sputum and patients with smear-positive sputum for pulmonary tuberculosis.

\begin{tabular}{ccccc}
\hline & $\begin{array}{c}\text { Patients with } \\
\text { smear-negative sputum } \\
(\mathrm{n}=42)\end{array}$ & $\begin{array}{c}\text { Patients with } \\
\text { smear-positive sputum } \\
(\mathrm{n}=14)\end{array}$ & $\begin{array}{c}\text { Total } \\
(\mathrm{n}=56)\end{array}$ & $P$-value \\
\hline Sex & $17(40.5 \%)$ & $4(28.6 \%)$ & $21(37.5 \%)$ & 0.32 \\
Female & $25(59.5 \%)$ & $10(71.4)$ & $35(62.5)$ & \\
Male & $60.05 \pm 18.26$ & $65.86 \pm 16.87$ & & 0.30 \\
$\begin{array}{c}\text { Age, years } \\
\text { Culture-positive } \\
\text { sputum }\end{array}$ & $25(59.5 \%)$ & $14(100 \%)$ & $39(69.6 \%)$ & 0.003 \\
$\begin{array}{c}\text { Smear-positive } \\
\text { bronchial washing } \\
\begin{array}{c}\text { Culture-positive } \\
\text { bronchial washing }\end{array}\end{array}$ & $5(11.9 \%)$ & $7(50.0 \%)$ & $12(21.4 \%)$ & 0.006 \\
\hline
\end{tabular}

Table 2. Among patients with smear-negative sputum for pulmonary tuberculosis, comparison between patients with culture-negative sputum and patients with culture-positive sputum.

\begin{tabular}{ccccc}
\hline & $\begin{array}{c}\text { Patients with } \\
\text { culture-negative sputum } \\
(\mathrm{n}=17)\end{array}$ & $\begin{array}{c}\text { Patients with } \\
\text { culture-positive sputum } \\
(\mathrm{n}=25)\end{array}$ & $\begin{array}{c}\text { Total } \\
(\mathrm{n}=42)\end{array}$ & $P$-value \\
\hline $\begin{array}{c}\text { Smear-positive } \\
\text { bronchial washing }\end{array}$ & $2(11.8 \%)$ & $3(12 \%)$ & $5(11.9 \%)$ & 0.68 \\
$\begin{array}{c}\text { Culture-positive } \\
\text { bronchial washing }\end{array}$ & $17(100 \%)$ & $19(76 \%)$ & $36(85.7 \%)$ & 0.03 \\
\hline
\end{tabular}

\section{Discussion}

In this study, the positive rates of cultures of both sputum and bronchial washing were higher in patients with smear-positive pulmonary TB compared to patients with SNPT. In a previous study, there were approximately $10^{4} / \mathrm{mL}$ tubercle bacilli in the sputum on microscopic examination [8]. This may be because there are more organisms in patients with smear-positive sputum. All patients with smear-positive pulmonary TB showed culture-positive sputum, but only 50\% showed smear-positive bronchial washing, and $85.7 \%$ showed culture-positive bronchial washing, exhibiting a significant difference. This may be because of the bacteriostatic effect of the local anesthetic xylocaine or delayed processing of the bronchial wash samples [5]. Bronchoalveolar lavage could possibly have a better diagnostic accuracy, but it is not frequently performed in resource-constrained settings.

In this study, $40.47 \%$ of the patients showed both smear- and culture-negative sputum, and they were diagnosed using the broncho washing specimen. However, only $11.9 \%$ were diagnosed based on smear-positive bronchial washing. The others were diagnosed based on culture-positive bronchial washing, and more 
than six weeks were needed to confirm the presence of $M . T B$. There was a limitation to the method of bronchoscopic washing of delay in diagnosis and control of the transmission of pulmonary TB.

The limitation of this study was the small sample size and lack of analysis of the accuracy of the diagnostic method.

\section{Conclusion}

In conclusion, considering the risk of transmission of $\mathrm{TB}$ to the operators of bronchoscopic washing and risks of the operation to the patients, it is difficult to determine its appropriate indication and timing. For patients with smear-negative sputum, it is necessary to perform bronchoscopic washing to raise the diagnostic rate of pulmonary TB. Further, in areas of the high prevalence of TB, it is necessary to use a fast and accurate examination method to control the transmission of pulmonary TB.

\section{Conflicts of Interest}

There is no conflict of interest regarding the publication of this paper.

\section{References}

[1] World Health Organization (2016) Global Tuberculosis Report 2016. World Health Organization, Geneva.

[2] Korea National Tuberculosis Association (2017) 7th Korea National Health and Nutrition Examination Survey 1st Year (2016) Tuberculin Survey Support and Quality Control. Korea Centers for Disease Control \& Prevention, Cheongju. http://www.ndsl.kr/ndsl/search/detail/report/reportSearchResultDetail.do?cn=TRK O201700005159

https://doi.org/10.21742/apjpc.2017.1.2.02

[3] WHO (2010) Treatment of Tuberculosis: Guidelines. 4th Edition. http://whqlibdoc.who.int/publications/2010/9789241547833_eng.pdf

[4] Soto, A., Agapito, J., Acuna-Villaorduna, C., Solari, L., Samalvides, F. and Gotuzzo, E. (2008) Evaluation of the Performance of Two Liquid-Phase Culture Media for the Diagnosis of Pulmonary Tuberculosis in a National Hospital in Lima, Peru. International Journal of Infectious Diseases, 13, 40-45. https://doi.org/10.1016/j.ijid.2008.03.023

[5] Mohan, A. and Sharma, S.K. (2008) Fibreoptic Bronchoscopy in the Diagnosis of Sputum Smear-Negative Pulmonary Tuberculosis: Current Status. Indian Journal of Chest Diseases and Allied Sciences, 50, 67-78.

[6] Feinsilver, S.H. and Fein, A.M. (1995) Textbook of Bronchoscopy. Lippincott Williams \& Wilkins, Baltimore.

[7] Kim, M.H., Suh, G.Y., Chung, M.P., Kim, H., Kwon, O.J., Lee, J.H., Lee, N.Y. and Koh, W.J. (2007) The Value of Routinely Culturing for Tuberculosis during Bronchoscopies in an Intermediate Tuberculosis-Burden Country. Yonsei Medical Journal, 48, 969-972. https://doi.org/10.3349/ymj.2007.48.6.969

[8] Kim, T.C., Blackman, R.S., Heatwole, K.M., Kim, L. and Rochester, D.F. (1984) Acid-Fast Bacilli in Sputum Smears of Patients with Tuberculosis. The American Review of Respiratory Disease, 129, 264-268. 